\title{
Dealing with COVID-19 in Medical Practice: Yet an Uncertain Situation
}

\author{
Prabin Shrestha $\boldsymbol{P h} \boldsymbol{D}^{\mathbf{1}}$ iD
}

${ }^{1}$ Department of Neurosciences, B\&B Hospital, Lalitpur, Nepal

Date of submission: $9^{\text {th }}$ November 2021

Date of acceptance: $18^{\text {th }}$ November 2021

Date of publication: $1^{\text {st }}$ December 2021

\section{Abstract}

COVID-19 pandemic is still uncertain and is going to last longer. The world has learnt a lot to fight against it. However, the world has to learn to live and deal with it in the days ahead. Medical practice has to be innovated and modified to protect medical professionals as well as patients.

Key words: COVID-19, Medical practice, Pandemic, Uncertain situation.

\section{Pandemic overview}

Tt has been almost 2 years since the evolution of Novel LCoronavirus 2019 (2019-nCoV), later named "Severe Acute Respiratory Syndrome Coronavirus 2" (SARS$\mathrm{CoV}-2$ ) and more than one and a half years since its outbreak as Coronavirus Disease of 2019 (COVID-19). ${ }^{1}$

Since WHO declared the COVID-19 pandemic on 11 March 2020, many waves have appeared and passed by in the world. The pandemic waves affected different geographical regions at different times. Pandemic waves do not appear at the same time in different regions and they appear repeatedly in the same area. Europe, Americas, including the United States of America (USA) and Latin America, and South Asia are amongst the worst hit regions so far. Europe has about 26\%, USA 19\% and South Asia $16 \%$ of world's total COVID-19 cases until November 2021. Similarly, Europe has about 26\%, USA $15 \%$ and South Asia about $12 \%$ of the world's total COVID-19 deaths. Pandemic waves have appeared in these regions

Access this article online
Website: https://www.nepjol.info/index.php/NJN
DOI: https://doi.org/10.3126/njn.v18i4.40757
HOW TO CITE
Shrestha P. Dealing with COVID-19 in Medical Practice: Yet an
Uncertain Situation. NJNS. 2021;18(4):1-3.

Address for correspondence:

Dr. Prabin Shrestha

Chief Neurosurgeon,

Department of Neurosciences,

B\&B Hospital,

Gwarko, Lalitpur, Nepal.

E-mail: prabinshrestha@hotmail.com

Phone: +977-9851079995

Copyright (C) 2021 Nepalese Society of Neurosurgeons (NESON)

ISSN: 1813-1948 (Print), 1813-1956 (Online)

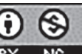

This work is licensed under a Creative Commons Attribution-Non Commercial 4.0 International License. repeatedly and it's not yet certain that when and how will be the next wave. ${ }^{2}$

In October-November 2021, several countries in Europe including UK, Russia, Turkey etc. had a new pandemic wave. Similarly, countries in Eastern Europe namely Slovenia, Serbia, Croatia, Ukraine etc. suffered not only a new pandemic wave that time but also had the biggest ever wave of daily new COVID-19 cases. Europe observed world's more than $55 \%-60 \%$ of daily new COVID-19 cases that time with almost the equal proportion of new deaths. Europe had, in contrary, minimal daily new cases in May-June 2021 occupying only about $10-12 \%$ of world's total new cases. ${ }^{1,2}$

In October-November 2021, South Asia, including India, seemed quite quiet with less than world's $5 \%$ daily new COVID-19 cases whereas it had world's more than half of daily new cases in May 2021. ${ }^{1,2}$

The Americas showed a relatively uniform pattern of pandemic curve without much peaks and falls unlike other regions. In October-November 2021, Latin America including Brazil had minimal number of daily new case whereas in the beginning of 2021 there was more than half of world's new cases. ${ }^{1,2}$

This shows pandemic waves are not uniform around the world at a point of time.

Even after such a long time of pandemic outset, newer and even bigger pandemic waves are appearing. Despite the achievement of knowledge and technical advancement about COVID-19 many more newer variants and cases have been appearing.

Development of the vaccine against SARS-CoV-2 and its widespread use in a short span of time is a breakthrough of this century. In the past less than 1-year time, highly developed countries such as Singapore, UAE (United Arab Emirates), UK (United Kingdom), Canada, South Korea, Japan etc. have already fully vaccinated more than $75 \%$ of their people till November $2021 .^{3}$ UAE has fully vaccinated the highest proportion of its population in the world, about $87 \%$, whereas India has fully vaccinated the 


\section{Shrestha et al}

highest number of people, about 340 million. America has fully vaccinated about $57 \%$ of its people whereas Nepal and India have fully vaccinated about $25 \%$. Europe has most widely covered its people with vaccine followed by Americas. ${ }^{3,4}$

Few vaccines have been approved even for the children aged between 12-18 years and thus many children in Europe and Americas have been immunized against COVID-19. Booster shots or the $3^{\text {rd }}$ dose of anti SARSCoV-2 vaccines have also been made available by some companies such as Pfizer, Moderna, Johnson and Johnson etc. for elderly above 65 years old, those with secondary and chronic medical conditions and those who live in long-term care settings. ${ }^{5}$ CDC (Center for Disease Control and Prevention) has recommended vaccination for small children, 5 years or older, and Pfizer has already started it in some states of America.

Despite such a huge advancement and achievement in the field of COVID-19, the number of daily new cases has not yet decreased significantly. Rather the biggest waves have been appearing around the world.

The above facts suggest that COVID-19 pandemic is not yet well controlled and it is still in an uncertain situation. It is still uncertain when and how the current pandemic will end. Thus, we have to learn to live and deal with COVID-19, only fighting against it is not sufficient.

Medical practice and medical education are the most hampered fields in the pandemic period. Likewise, health care professionals have been at the highest risk in this period. In such a scenario the medical practice, including neurosurgical one, has to be re-innovated to a more appropriate way.

\section{Measures to be taken}

Even though, public has become much aware of COVID-19 and its pandemic, they may not yet be aware of how they should present themselves in the setup of medical services and hospitals. Moreover, various kinds of medical centers and hospitals may have different policies of screening visitors before they enter the center. Therefore, all of them need to make a uniform protocol of screening visitors and management of patients in emergency and inpatient departments. People must learn to avoid unnecessary crowds to save not only themselves but others too. Strict distancing and using masks are simple yet most effective measures of preventing infection transmission. Daily activities ranging from simple behavioral changes to the use of highly advanced techniques, should be implemented to deal with COVID-19 pandemic.

Following precautions and measures can be of help, in the context of Nepal, to minimize the spread of COVID- 19 .

A simple measure such as measurement of temperature and peripheral oxygen saturation of everybody entering the hospital will be of great help to screen the crowd of people. Anybody suspected should be prohibited to enter the hospital and should be directed to COVID-19 clinic.

Similarly, an online appointment system for any kind of medical service, if possible, not only helps to screen COVID-19 infection before visiting a medical center, but also provides adequate information and guidelines to the public that he or she should follow in the hospital setup. The same thing should be applied to those who accompany the patient or a client also. It will minimize the crowd as well.

Emergency surgery should be avoided as far as possible, which is not only risky for the surgical team but also costlier to the patient. Elective surgery should be encouraged and can be planned by fixing the date only one to two weeks later. In this period patients can take adequate precaution and restrict him/herself to avoid catching the infection in addition to performing corona screening and other pre-operative investigations. This can increase the safety margin to both the surgical team and the patient.

Routine and periodic self-isolation of medical practitioners, health care providers, frontline workers and other professionals is being practiced in many countries. Isolating oneself for about one week every few months from his/her regular work during the pandemic period is an effective way of avoiding catching and preventing transmission of infection. It helps to control the crowd in the working setup and allows refreshing him/herself physically and mentally. ${ }^{5}$

Telemedicine is not a new technology but needs to be promoted further during the corona crisis. This helps in avoiding unnecessary crowding of people in the medical centers. Public awareness activities need to focus on this aspect, especially in a less educated and ignorant society like ours. However, its financial, technical and legal aspects need proper management and implementation. ${ }^{6}$

Webinars and other online medical educational and academic activities have become a routine part of all medical practitioners' life during the pandemic period. It is very helpful for two-way communication and for observational activities as well. It has to be continued as long as the threat of pandemic exists.

Many European and American countries have started so-called "Drive Thru" services for many simple medical services. Patient goes to a medical center, stops his/her car in front of a shield window and communicates with the staff with a microphone for the service. Self-nasal swab collection for PCR, dispensing medicines etc. are few examples of this service.

In summary, COVID-19 pandemic is still uncertain and is going to last longer. The world has learnt a lot to fight against it. However, the world has to learn to live and 
deal with it in the days ahead. Medical practice has to be innovated and modified to protect medical professionals as well as patients.

\section{Conflict of Interest: None Source(s) of support: None}

\section{References}

1. World Health Organization. Coronavirus (COVID -19) Dashboard; 2021 November 8. Available from: https://covid19. who.int/?gclid=EAIaIQobChMIpaX.

2. Worldometer. Coronavirus Worldwide Graphs; 2021 November 8. Available from: https://www. worldometers.info/coronavirus/worldwide-graphs/

3. Our World in Data. Coronavirus (COVID-19) Vaccinations; 2021 November 8. Available from: https://ourworldindata.org/covid-vaccinations
4. Government of Nepal. Health Sector Response to COVID-19; 2021 November 9. Available from: https://mohp.gov.np/attachments/article/703/ Responding\%20to\%20COVID-19,\%20Health\%20 sector $\% 20 \%$ preparedness, $\% 20$ response $\% 20$ and $\% 20$ lessons\%20learnt.pdf

5. Centers for Disease Control and Prevention. Some COVID-19 Vaccine Recipients Can Get Booster Shots; 2021 November 9. Available from: https:// www.cdc.gov/coronavirus/2019-ncov/vaccines/ booster-shot.html

6. American Medical Association. COVID-19: A physician guide to keeping your practice open; 2020 December 16. Available from https:/www.amaassn.org/delivering-care/public-health/covid-19physician-guide-keeping-your-practice-open

7. Shen YT, Chen L, Yue WW, Xu HX. Digital Technology-Based Telemedicine for the COVID-19 Pandemic. Front Med (Lausanne). 2021; 8: 646506. https://doi.org/10.3389/fmed.2021.646506 